\title{
The Existence and Stability of Solutions for Vector Quasiequilibrium Problems in Topological Order Spaces
}

\author{
Qi-Qing Song \\ College of Science, Guilin University of Technology, Guilin 541004, China \\ Correspondence should be addressed to Qi-Qing Song; songqiqing@gmail.com
}

Received 4 June 2013; Accepted 18 July 2013

Academic Editor: Wei-Shih Du

Copyright (C) 2013 Qi-Qing Song. This is an open access article distributed under the Creative Commons Attribution License, which permits unrestricted use, distribution, and reproduction in any medium, provided the original work is properly cited.

In a topological sup-semilattice, we established a new existence result for vector quasiequilibrium problems. By the analysis of essential stabilities of maximal elements in a topological sup-semilattice, we prove that for solutions of each vector quasi-equilibrium problem, there exists a connected minimal essential set which can resist the perturbation of the vector quasi-equilibrium problem.

\section{Introduction}

Vector equilibrium problems can unify many nonlinear problems such as vector optimization, vector variational inequality [1], and vector complementarity problems [2]. Recently, not only vector equilibrium problems [3-7] but also vector quasiequilibrium problems [8-14] and the system of vector quasi-equilibrium problems have attracted much attention [15-19].

Topological vector spaces provide the usual mathematical framework in the study of many problems. To avoid the linear feature, sup-semilattices may be good choices. In fact, some results like the existence of KKM points in topological spaces were established in topological sup-semilattices [20], where a two-tuple $(X, \leq)$ is said to be a sup-semilattice, if $X$ is a partially ordered set with the partial ordering $\leq$, in which every pair $\left(x, x^{\prime}\right)$ has a least upper bound $x \vee x^{\prime}$.

The aim of this paper is to study the existence and essential stability of vector quasi-equilibrium problems in topological sup-semilattices. In order to achieve this, firstly, we give a stability result in relation to maximal elements in a topological sup-semilattice. Secondly, a new existence result for vector quasi-equilibrium problems is established, and we show that each vector quasi-equilibrium problem has a connected minimal essential set in its solution set.

\section{Preliminaries}

Let $(X, \leq)$ be a sup-semilattice. If $x$ and $x^{\prime}$ are two elements in $(X, \leq)$ and $x \leq x^{\prime}$, the set $\left[x, x^{\prime}\right]=\left\{y \in X: x \leq y \leq x^{\prime}\right\}$ is called an order interval. Let $A, A^{\prime}$ be two nonempty finite subsets of $X$. Then the set $\Delta A=\cup_{x \in A}[x$, sup $A]$ is well defined and has the properties: $A \subseteq \Delta A$ and $\Delta A \subseteq \Delta A^{\prime}$ if $A \subseteq A^{\prime}$.

Definition 1 (see [20]). A subset $E \subseteq X$ is $\Delta$-convex, if for any nonempty finite subset $A \subseteq E$, we have $\Delta A \subseteq E$. $E$ being a $\Delta$-convex set is equivalent to the following conditions:

(a) if $x, x^{\prime} \in E$, then its least upper bound $x \vee x^{\prime} \in E$.

(b) if $x, x^{\prime} \in E$ and $x \leq x^{\prime}$, then the order interval $\left[x, x^{\prime}\right] \subset E$.

It is easy to check that the intersection of two $\Delta$-convex sets is $\Delta$-convex as well.

A topological space $X$ is said to be a topological supsemilattice if $X$ is equipped with a sup-semilattice as its partial ordering denoted by $\leq$, for which $f: X \times X \rightarrow X$ with $\left(x, x^{\prime}\right) \rightarrow x \vee x^{\prime}$ is a continuous function.

Let $Y$ be a topological vector space and $\theta$ the zero element in $Y$. A subset $C \subset Y$ is called a cone if, for any $y \in C$ and real number $t>0$, ty $\in C$. A cone $C$ is convex if $C$ is a convex set. If $C \cap-C=\{\theta\}$, it is called a pointed cone.

Definition 2 (see [18]). Let $X$ be a topological sup-semilattice, $Y$ a topological vector space with a cone $C \subset Y, \varphi: X \rightarrow Y$ a vector-valued function.

(a) $\varphi: X \rightarrow Y$ is $C_{\Delta}$-quasiconcave if, for any nonempty two points subset $A=\left\{x_{1}, x_{2}\right\} \subset X$ and $y \in Y, \varphi(A) \subset$ $y+C \Rightarrow \varphi(\Delta A) \subset y+C$. 
(b) $\varphi: X \rightarrow Y$ is said to be $C_{\Delta}$-quasiconcave-like if, for any $x_{1}, x_{2} \in X, \varphi\left(\Delta\left\{x_{1}, x_{2}\right\}\right) \in \varphi\left(x_{1}\right)+C$ or $\varphi\left(\Delta\left\{x_{1}, x_{2}\right\}\right) \in \varphi\left(x_{2}\right)+C$.

Remark 3. In general cases, $C_{\Delta}$-quasiconcave, $C_{\Delta}$-quasiconcave-like, and usual quasiconcave functions are independent of each other. See examples in [18]. Let $\varphi: \mathbb{R} \rightarrow \mathbb{R}$, $C=-\mathbb{R}^{+}$. Then the partial order on $\mathbb{R}$ is " $\leq$ " (less than or equal to); hence, the $C_{\Delta}$-quasiconcave, $C_{\Delta}$-quasiconcavelike, and usual quasiconcave property of $\varphi$ coincide (the usual quasiconcave function $\varphi$ means that for any $x_{1}, x_{2}, y \in \mathbb{R}$, $\varphi\left(x_{1}\right) \leq y$ and $\varphi\left(x_{2}\right) \leq y \Rightarrow \varphi\left(\lambda x_{1}+(1-\lambda) x_{2}\right) \leq y$, for all $\lambda \in[0,1])$.

Lemma 4 (see [18]). Let $X$ be a topological sup-semilattice, $Y$ a Hausdorfflocally convex topological vector space with a closed, convex, and pointed cone $C \subset Y$. If the vector-valued function $\varphi: X \rightarrow Y$ is $C_{\Delta}$-quasiconcave or $C_{\Delta}$-quasiconcave-like, then the set $A=\{x: \varphi(x) \in \operatorname{int} C\}$ is $\Delta$-convex.

Now we introduce the vector quasiequilibrium problem (VQEP) that we will consider in this paper.

Let $X$ be a topological sup-semilattice and $Y$ a topological vector space. $C \subset Y$ is a closed, convex, and pointed cone with int $C \neq \emptyset . \varphi: X \times X \rightarrow Y$ is a vector-valued function, and $G$ is a multivalued mapping on $X$. The vector quasi-equilibrium problem $\phi$ with $\phi=(X, Y, C, \varphi, G)$ is to find $\bar{x} \in X$, such that

$$
\bar{x} \in G(\bar{x}): \varphi(\bar{x}, y) \notin \operatorname{int} C, \quad \forall y \in G(\bar{x}) .
$$

Let $G(x)=X$, for all $x \in X$; then the VQEP is just a vector equilibrium problem $(X, Y, C, \varphi)(\mathrm{VEP})$. That is to find $\bar{x} \in X$, such that

$$
\varphi(\bar{x}, y) \notin \operatorname{int} C, \quad \forall y \in X .
$$

Definition 5 (see $[21,22]$ ). A vector-valued function $\varphi: X \rightarrow$ $Y$ is said to be C-continuous on $X$ if, for each $x \in X$ and any open neighborhood $V(\theta)$ of $\theta$ in $Y$, there exists an open neighborhood $O(x)$ of $x$ in $X$ such that

$$
\forall x^{\prime} \in O(x), \quad \varphi\left(x^{\prime}\right) \in \varphi(x)+V(\theta)+C .
$$

Remark 6. For a function $\varphi: X \rightarrow \mathbb{R}, \varphi$ is $\mathbb{R}_{+}$-continuous on $X$ if and only if $\varphi$ is lower semicontinuous on $X$.

A maximal element version of the Browder fixed point theorem in a topological sup-semilattice can be found in [18]. We limit it in a metric space as the following lemma.

Lemma 7 (see [18]). Let $(X, d)$ be a compact sup-semilattice with path connected interval, where $d$ is the metric on $X$, $S: X \rightarrow 2^{X}$ a multivalued map on $X$ with the conditions: (i) for all $x \in X, S(x)$ is $\Delta$-convex; (ii) for all $y \in$ $X, S^{-1}(y)=\{x \in X: y \in S(x)\}$ is open in $X$; (iii) for all $x \in$ $X, x \notin S(x)$. Then there exists an $\bar{x} \in X$, such that $S(\bar{x})=\emptyset$.

Remark 8. The existence of a metric space with a supsemilattice can be guaranteed. For instance, let $x^{j}=$ $\left(x_{1}^{j}, \ldots, x_{i}^{j}, \ldots, x_{n}^{j}\right) \in \mathbb{R}^{n}, j=1,2$, if $x^{1} \leq x^{2}$ means that $x^{2} \in$
$x^{1}+\mathbb{R}_{+}^{n}$, then $x^{1} \vee x^{2}=\bar{x}$, where $\bar{x}_{i}=\max \left\{x_{i}^{1}, x_{i}^{2}\right\}$. Clearly, $\left(\mathbb{R}^{n}, \leq\right)$ with the usual Euclidean metric is a topological supsemilattice.

Let $M$ denote the collection of $S$ satisfying all the conditions of Lemma 7. For any $S_{1}, S_{2} \in M$, define the metric between $S_{1}$ and $S_{2}$ as

$$
\rho\left(S_{1}, S_{2}\right)=\sup _{y \in X} h\left(X \backslash S_{1}^{-1}(y), X \backslash S_{2}^{-1}(y)\right),
$$

where $h$ is the Hausdorff metric induced by $d$. For each $S \in M$ and each $y \in X$, since $y \notin S(y)$, we have $y \in X \backslash S^{-1}(y)$, that is, $X \backslash S^{-1}(y) \neq \emptyset$. Noting that $X \backslash S^{-1}(y)$ is closed, the metric $\rho$ on $M$ is well defined. Then $(M, \rho)$ is a metric space.

For each $S \in M$, denote by $F(S)$ the set of all maximal elements of $S$. Then $F$ defines a multivalued mapping from $M$ to $X$ and $F(S)=\cap_{y \in X}\left(X \backslash S^{-1}(y)\right)$.

Definition 9. For each $S \in M$, a set $e(S)$ is called an essential set of $F(S)$ if it satisfies the following conditions:

(1) $e(S)$ is closed subset of $F(S)$.

(2) For any open set $U \supset e(S)$, there exists an open neighborhood $O(S)$ of $S \in M$ such that $U \cap F\left(S^{\prime}\right) \neq \emptyset$, for any $S^{\prime} \in O(S)$.

A set $m(S)$ is called a minimal essential set of $F(S)$ if it is a minimal element of all essential sets ordered by set inclusion in $F(S)$. A connected component in $F(S)$ is called an essential component, if it includes at least one minimal essential set of $F(S)$.

We recall some notions about multi-valued mappings. Let $G: Y \rightarrow 2^{P}$ be a multi-valued mapping, where $Y, P$ are two topological vector spaces. Then (i) $G$ is said to be upper semicontinuous at $y \in Y$, if for each open set $U \supset G(y)$, there exists an open neighborhood $O(y)$ of $y$ such that $U$ ว $G\left(y^{\prime}\right)$ for any $y^{\prime} \in O(y)$. (ii) $G$ is lower semi-continuous at $y \in Y$, if for each open set $U \cap G(y) \neq \phi$, there exists an open neighborhood $O(y)$ of $y$ such that $U \cap G\left(y^{\prime}\right) \neq \phi$ for any $y^{\prime} \in$ $O(y)$.

Remark 10. For each $S \in M$, a set $e(S) \subset F(S)$ is essential if $F$ is lower semi-continuous at $S$. If $F$ is upper semi-continuous at $S$, then $F(S)$ itself is an essential set. For any two closed sets $A, B \subset F(S)$ with $A \subset B$, if $A$ is essential, then $B$ is also essential. For each $S \in M$ and each $y \in X$, if $S^{-1}(y)$ is open, then $X \backslash S^{-1}(y)$ is closed; hence, $F(S)$ is closed because we have $F(S)=\cap_{y \in X} X \backslash S^{-1}(y)$; consequently, $F(S)$ is compact.

Lemma 11 (see [23]). Let $(X, d)$ be a metric space, $K_{1}$ and $K_{2}$ two nonempty compact subsets of $X, U_{1}$, and $U_{2}$ two nonempty disjoint open subsets of $X$. If $h\left(K_{1}, K_{2}\right)<d\left(U_{1}, U_{2}\right)$, then $h\left(K_{1},\left(K_{1} \backslash U_{2}\right) \cup\left(K_{2} \backslash U_{1}\right)\right) \leq h\left(K_{1}, K_{2}\right)$, where $h$ is the Hausdorff metric defined on $X$. 


\section{The Stability of Maximal Elements on Topological Semilattices}

Theorem 12. $F: M \rightarrow 2^{X}$ is an upper semi-continuous mapping with compact values.

Proof. For each $S \in M$, by Remark 10, $F(S)$ is compact. Suppose that $F$ is not upper semi-continuous. Then there is a $S \in M$, an open set $U$ with $U \supset F(S)$ and $S_{n}$, such that $S_{n} \rightarrow S$ and $F\left(S_{n}\right) \not \subset U, n=1,2, \ldots$. That is, there exists a point $x_{n} \in F\left(S_{n}\right)$ such that $x_{n} \notin U$. Without loss of generality, we may assume that $x_{n} \rightarrow x^{*}$. Since $S_{n} \rightarrow S$, it holds that $X \backslash S_{n}^{-1}(y) \rightarrow X \backslash S^{-1}(y)$, for all $y \in X$. Since $x_{n} \in F\left(S_{n}\right)$, we have $x_{n} \in X \backslash S_{n}^{-1}(y)$, for all $y \in X$. As $n$ gets close to infinity, we can obtain that $x^{*} \in X \backslash S^{-1}(y)$, for all $y \in X$, that is, $x^{*} \in F(S) \subset U$. This results in the fact that $x_{n} \in U$ while $n$ is large enough, a contradiction with $x_{n} \notin U$. Therefore, $F$ is definitely upper semi-continuous.

Theorem 13. For each $S \in M$, there exists at least a minimal essential set of $F(S)$. If $m(S)$ is a minimal essential set of $F(S)$, then $m(S)$ is connected.

Proof. For the existence, by Remark 10, each decreasing chain, consisting of essential subsets of $F(S)$, has a minimal element, which is the intersection of the chain. By the Zorn's lemma, the minimal element is just a minimal essential set. For the connectedness, by way of contradiction, suppose that $m(S)$ is not connected. There exist two disjoint closed sets $C_{1}(S), C_{2}(S)$ such that $m(S)=C_{1}(S) \cup C_{2}(S)$.

Since $C_{i}(S)$ is not essential, there is an open set $W_{i}$ with $W_{i} \supset C_{i}(S)$ such that for any $\varepsilon>0$, there exists a $S_{i} \in M$ with $\rho\left(S, S_{i}\right)<\varepsilon$ and $F\left(S_{i}\right) \cap W_{i}=\emptyset, i=1,2$. Clearly, $C_{i}(S)$ is compact, then there is an open set $U_{i}$ with $C_{i}(S) \subset U_{i} \subset$ $W_{i}, i=1,2$, such that $U_{1} \cap U_{2}=\emptyset$. For $U_{1} \cup U_{2} \supset m(S)$, because $m(S)$ is essential, there is a number $\delta<2 d\left(U_{1}, U_{2}\right)$, such that $F(T) \cap\left(U_{1} \cup U_{2}\right) \neq \emptyset$ for each $T$ satisfying $\rho(T, S)<\delta$. Therefore, we can select a $S_{i} \in M$ such that $\rho\left(S, S_{i}\right)<\delta / 4$ and $F\left(S_{i}\right) \cap U_{i}=\emptyset, i=1,2$. Then $\rho\left(S_{1}, S_{2}\right)<\rho\left(S_{1}, S\right)+\rho\left(S, S_{2}\right)<$ $\delta / 2<d\left(U_{1}, U_{2}\right)$.

Define a multi-valued mapping $S^{\prime}: M \rightarrow 2^{X}$ as

$$
S^{\prime}= \begin{cases}S_{1}(x), & x \in U_{1}, \\ S_{2}(x), & x \in U_{2}, \\ S_{1}(x) \cap S_{2}(x), & x \in X \backslash\left(U_{1} \cup U_{2}\right) .\end{cases}
$$

We show that $S^{\prime} \in M$.

(a) For each $x \in X$, since $x \notin S_{1}(x)$ and $x \notin S_{2}(x)$, we have $x \notin S^{\prime}(x)$;

(b) for each $x \in X$, because $S_{1}(x)$ and $S_{2}(x)$ are $\Delta$-convex sets, it follows that $S^{\prime}(x)$ is $\Delta$-convex;

(c) for each $y \in X$, we have

$$
\begin{aligned}
& S^{\prime^{-1}}(y) \\
& \quad=\left(S_{1}^{-1}(y) \cap S_{2}^{-1}(y)\right) \cup\left(S_{2}^{-1}(y) \cap U_{2}\right) \cup\left(S_{1}^{-1}(y) \cap U_{1}\right) .
\end{aligned}
$$

Noting that $S_{1}^{-1}(y), S_{2}^{-1}(y), U_{1}$, and $U_{2}$ are open sets, it follows that $S^{\prime-1}(y)$ is open.

Through a direct calculation, $X \backslash S^{\prime-1}(y)$ can be written as

$$
\begin{aligned}
\left(\left(X \backslash S_{1}^{-1}(y)\right) \cap\left(X \backslash S_{2}^{-1}(y)\right)\right) \\
\cup\left(\left(X \backslash S_{1}^{-1}(y)\right) \cap\left(X \backslash U_{2}\right)\right) \\
\cup\left(\left(X \backslash S_{2}^{-1}(y)\right) \cap\left(X \backslash U_{1}\right)\right) .
\end{aligned}
$$

Take any $x \in X \backslash S^{\prime-1}(y)$. Note that if $x \in U_{1}$, then $x \in$ $X \backslash U_{2}$; if $x \in U_{2}$, then $x \in X \backslash U_{1}$; if $x \in X \backslash\left(U_{1} \cup U_{2}\right)$, then $x \in\left(X \backslash U_{1}\right) \cap\left(X \backslash U_{2}\right)$. Consequently, we can obtain that if $x \in\left(X \backslash S_{1}^{-1}(y)\right) \cap\left(X \backslash S_{2}^{-1}(y)\right)$, then

$$
\begin{aligned}
x \in( & \left.\left(X \backslash S_{1}^{-1}(y)\right) \cap\left(X \backslash U_{2}\right)\right) \\
& \cup\left(\left(X \backslash S_{2}^{-1}(y)\right) \cap\left(X \backslash U_{1}\right)\right) .
\end{aligned}
$$

Therefore, we have

$$
\begin{aligned}
X \backslash S^{\prime-1} & (y) \\
= & \left(\left(X \backslash S_{1}^{-1}(y)\right) \cap\left(X \backslash U_{2}\right)\right) \\
& \cup\left(\left(X \backslash S_{2}^{-1}(y)\right) \cap\left(X \backslash U_{1}\right)\right) .
\end{aligned}
$$

Since $\rho\left(S_{1}, S_{2}\right)<d\left(U_{1}, U_{2}\right)$, by Lemma 11, we have

$$
\begin{aligned}
h( & \left.X \backslash S^{\prime^{-1}}(y), X \backslash S_{1}^{-1}(y)\right) \\
& \leq h\left(X \backslash S_{2}^{-1}(y), X \backslash S_{1}^{-1}(y)\right) \\
& \leq \rho\left(S_{2}, S\right)+\rho\left(S, S_{1}\right)<\frac{\delta}{2} .
\end{aligned}
$$

That is, $\rho\left(S^{\prime}, S_{1}\right)<\delta / 2$. This results in the fact that

$$
\rho\left(S^{\prime}, S\right)<\rho\left(S^{\prime}, S_{1}\right)+\rho\left(S_{1}, S\right)<\delta .
$$

Consequently, we have $F\left(S^{\prime}\right) \cap\left(U_{1} \cup U_{2}\right) \neq \emptyset$. If there is a point $x \in U_{i} \cap F\left(S^{\prime}\right)$, then $x \in U_{i}$ and $x \in X \backslash S_{i}^{-1}(y)$, for all $y \in X$, that is, $x \in U_{i}$ and $x \in F\left(S_{i}\right)$ which contradicts with $F\left(S_{i}\right) \cap$ $U_{i}=\emptyset, i=1,2$. Therefore, $m(S)$ is connected.

\section{The Existence and Stability of Solutions for VQEP}

This section gives an existence result in relation to VQEP in topological sup-semilattices and induces the existence of minimal essentially stable sets for each VQEP in the set of its solutions.

Theorem 14. Let $\phi=(X, Y, C, \varphi, G)$ be a VQEP, where $X$ is a compact topological sup-semilattice with path connected intervals, $Y$ is a Hausdorff locally convex topological vector space, and $G: X \rightarrow 2^{X}$ is a multi-valued mapping with nonempty and $\triangle$-convex values. If the VQEP satisfies that 
(i) for all $x \in X, \varphi(x, x) \notin \operatorname{int} C$;

(ii) for all $y \in X, x \rightarrow \varphi(x, y)$ is C-continuous;

(iii) for all $x \in X, y \rightarrow \varphi(x, y)$ is $C_{\Delta}$-quasiconcave-like or $C_{\Delta}$-quasiconcave;

(iv) for all $y \in X,\{x \in X: y \in G(x)\}$ is open in $X$,

(v) $\{x \in X: x \in G(x)\}$ is closed in $X$,

then the VQEP has a solution.

Proof. Denote $\{x \in X: x \in G(x)\}$ by $K$. Let $B: X \rightarrow 2^{X}$ such that $B(x)=\{y \in X: \varphi(x, y) \in \operatorname{int} C\}$, for all $x \in X$. Define

$$
S(x)= \begin{cases}B(x) \cap G(x), & x \in K, \\ G(x), & x \in X \backslash K .\end{cases}
$$

Then for each $x \in X$, if $x \in K$, we have $S(x)=B(x) \cap G(x)$, by the condition (i), $x \notin B(x)$, hence, $x \notin S(x)$; if $x \in X \backslash K$, from the definition of $K$, we have $x \notin G(x)=S(x)$.

Since $y \rightarrow \varphi(x, y)$ is $C_{\Delta^{-}}$-quasiconcave-like or $C_{\Delta^{-}}$ quasiconcave, by Lemma 4 , we have that $B(x)$ is $\Delta$-convex. Then $B(x) \cap G(x)$ is a $\Delta$-convex set, noting that $G$ has $\Delta$ convex values, we have that $S(x)$ is also $\Delta$-convex.

For each $y \in X$, we can check that

$$
S^{-1}(y)=\left(B^{-1}(y) \cap G^{-1}(y)\right) \cup\left(G^{-1}(y) \cap(X \backslash K)\right) .
$$

Take a point $x \in B^{-1}(y)=\{x \in X: \varphi(x, y) \in \operatorname{int} C\}$, since int $C$ is open, there is an open set $V(\theta)$ such that $V(\theta)+$ $\varphi(x, y) \subset$ int $C$, then, by the condition (ii), there exists an open neighborhood $O(x)$ in $X$ such that for all $x^{\prime} \in O(x)$,

$$
\varphi\left(x^{\prime}, y\right) \in \varphi(x, y)+V(\theta)+C \subset \operatorname{int} C+C \subset \operatorname{int} C .
$$

That is, $O(x) \subset B^{-1}(y)$; hence, $B^{-1}(y)$ is open. Noting that $X \backslash K$ and $G^{-1}(y)$ are open sets in $X$. We can obtain that $S^{-1}(y)$ is also open in $X$.

Thus, there is an $\bar{x} \in X$ such that $S(\bar{x})=\emptyset$ by Lemma 7. If $\bar{x} \in X \backslash K$, then $G(\bar{x})=\emptyset$, a contradiction to the fact that $G$ has nonempty values. Therefore, $\bar{x} \in K$ and $B(\bar{x}) \cap G(\bar{x})=\emptyset$, that is, $\bar{x} \in G(\bar{x}), \varphi(\bar{x}, y) \notin \operatorname{int} C$, for all $y \in X$.

By Theorem 14 and its proof, we can also obtain the existence result for VQEP as the following.

Corollary 15. Let $\phi=(X, Y, C, \varphi, G)$ be a VQEP, where $X$ is a compact topological sup-semilattice with path connected intervals, $Y$ is a Hausdorff topological vector space, and $G$ : $X \rightarrow 2^{X}$ is a multi-valued mapping with nonempty and $\Delta$ convex values. If the VQEP satisfies that

(i) for all $x \in X, \varphi(x, x) \notin \operatorname{int} C$;

(ii) for all $y \in X,\{x \in X: \varphi(x, y) \in \operatorname{int} C\}$ is open in $X$;

(iii) for all $x \in X,\{y \in X: \varphi(x, y) \in \operatorname{int} C\}$ is $\Delta$-convex;

(iv) for all $y \in X,\{x \in X: y \in G(x)\}$ is open in $X$,

(v) $\{x \in X: x \in G(x)\}$ is closed in $X$,

then the VQEP has a solution.
By Theorem 14, for the special case of VQEP without the feasible mapping $G$, we can obtain the existence result concerning VEP as the following.

Corollary 16. Let $(X, Y, C, \varphi)$ be a vector equilibrium problem, where $X$ is a compact topological sup-semilattice with path connected intervals, $Y$ is a Hausdorfflocally convex topological vector space. If the VEP satisfies the following conditions:

(i) for all $x \in X, y \rightarrow \varphi(x, y)$ is $C_{\Delta^{-}}$quasiconcave or $C_{\Delta^{-}}$ quasiconcave-like;

(ii) for all $y \in X, x \rightarrow \varphi(x, y)$ is C-continuous;

(iii) for all $x \in X, \varphi(x, x) \notin \operatorname{int} C$,

then this VEP has a solution.

Example 17. Let $X=[0,1] \times[0,1] \subset \mathbb{R}^{2}, C=-\mathbb{R}_{+}$. The $(X, \leq)$ is a sup-semilattice, in which $x^{1} \leq x^{2}$ means that $x^{2} \in$ $x^{1}+\mathbb{R}_{+}^{2}$, for all $x^{1}, x^{2} \in X$.

(a) For any $x=\left(x_{1}, x_{2}\right), y=\left(y_{1}, y_{2}\right) \in X$, the function $\varphi$ is defined as

$$
\varphi(x, y)=\left(1-y_{1}\right)\left(1-y_{2}\right)-\left(1-x_{1}\right)\left(1-x_{2}\right) .
$$

It can be easily checked that for each $x \in X, \varphi(x, \cdot)$ is $C_{\Delta}$ quasiconcave and $C_{\Delta}$ quasiconcave-like but not a usual quasiconcave function.

Denote by $D$ the set $(1 \times[0,1]) \cup([0,1] \times 1)$. For each $x=\left(x_{1}, x_{2}\right) \in X$, the multi-valued mapping $G$ satisfies that

$$
G(x)= \begin{cases}\left(x_{1}, 1\right] \times[0,1] \cup[0,1] \times\left(x_{2}, 1\right], & x \in X \backslash D, \\ (1,1), & x \in D .\end{cases}
$$

Note that $G$ is not a usual convex but a $\Delta$-convex multi-valued mapping. For each $y=\left(y_{1}, y_{2}\right) \in X$, if $y \in X \backslash(1,1)$, then $G^{-1}(y)=\left[0, y_{1}\right) \times\left[0, y_{2}\right)$; if $y=(1,1)$, then $G^{-1}(y)=X$. Thus, $G^{-1}(y)$ is open in $X$ for each $y \in X$. Then $\varphi$ and $G$ satisfy all the conditions in Theorem 14 . We can find that $\bar{x}=(1,1)$ is the unique solution for the VQEP, $(X, \mathbb{R}, C, \varphi, G)$.

(b) For any $x=\left(x_{1}, x_{2}\right), y=\left(y_{1}, y_{2}\right) \in X$, let $T(x)=B_{\varepsilon}(x)$, where $B_{\varepsilon}(x)$ is the $\varepsilon$-neighborhood of $x$ in $X$,

$$
\varphi(x, y)=\left(1+x_{1}-y_{1}\right)\left(1+x_{2}-y_{2}\right)
$$

and $G$ is the same as the setting in (a). Then the function $\varphi$ and the mappings $G$ and $T$ meet all the conditions in Theorem 14 . The set of solutions for the $\operatorname{VEP}(X, \mathbb{R}, C, \varphi)$ is the overall $X$ which is also the set of solutions for $(X, \mathbb{R}, C, \varphi, T)$. The solution of $(X, \mathbb{R}, C, \varphi, G)$ is just one point $\bar{x}=(1,1)$.

To study the stability of vector quasi-equilibrium problems, let $(X, d)$ be a metric space and define the set $M^{\prime}$ as

$$
\begin{aligned}
M^{\prime}=\{\phi & =(X, Y, C, \varphi, G): \\
& \quad \phi \text { satisfies all the conditions in Theorem } 14\}
\end{aligned}
$$

For each $\phi \in M^{\prime}$, by the proof of Theorem 14, we can find that a point $\bar{x} \in X$ is a solution of $\phi$ if and only if $\bar{x}$ is a maximal element of $S$ defined in the proof. Let $F^{\prime}(\phi)$ denote 
all the solutions of $\phi$. Then $F^{\prime}$ is a multi-valued mapping from $M^{\prime}$ to $X$. For any two $\phi_{1}, \phi_{2}$, define the metric $\rho^{\prime}\left(\phi_{1}, \phi_{2}\right)$ between $\phi_{1}$ and $\phi_{2}$ as

$$
\rho^{\prime}\left(\phi_{1}, \phi_{2}\right)=\rho\left(S_{1}, S_{2}\right),
$$

where $S_{1}$ and $S_{2}$ are multi-valued mappings corresponding to $\phi_{1}$ and $\phi_{2}$ in the proof of Theorem 14. Then $\left(M^{\prime}, \rho^{\prime}\right)$ is a metric space. Instead of $M, S$ and $F(S)$ by $M^{\prime}, \phi$ and $F^{\prime}(\phi)$ in Definition 9, we can also define essential sets $e(\phi)$, minimal essential sets $m(\phi)$ of $F^{\prime}(\phi)$, and essential component in $F^{\prime}(\phi)$. If an essential set $e(\phi)$ is singleton set $\left\{x^{*}\right\}, x^{*}$ is called an essential solution of $\phi$.

From Theorems 12 and 13, we have the following results.

Theorem 18. $F^{\prime}: M^{\prime} \rightarrow 2^{X}$ is an upper semi-continuous mapping with compact values. For each $\operatorname{VQEP} \phi \in M^{\prime}$, there exists at least a connected minimal essential set $m(\phi)$ of $F^{\prime}(\phi)$.

Remark 19. For each $\phi \in M^{\prime}, y \in X$, let

$$
A_{\phi}(y)=\{x: y \notin G(x) \text {, or } \varphi(x, y) \notin \operatorname{int} C \text { and } x \in G(x)\} \text {. }
$$

For any $\phi_{1}, \phi_{2} \in M^{\prime}$, from the definition of the metric between $S_{1}$ and $S_{2}$, then

$$
\rho^{\prime}\left(\phi_{1}, \phi_{2}\right)=\sup _{y \in X} h\left(A_{\phi_{1}}(y), A_{\phi_{2}}(y)\right),
$$

which gives an overall consideration of $\varphi$ and G. If $\phi_{1}$ and $\phi_{2}$ are two VEP, then

$$
\begin{aligned}
& \rho^{\prime}\left(\phi_{1}, \phi_{2}\right) \\
& =\sup _{y \in X} h\left(\left\{x: \varphi_{1}(x, y) \notin \operatorname{int} C\right\},\right. \\
& \left.\quad x\left\{x: \varphi_{2}(x, y) \notin \operatorname{int} C\right\}\right) .
\end{aligned}
$$

For the essential stability of solutions for VQEP, clearly, the class of perturbations induced by the metric $\rho^{\prime}$ is different from the perturbation of uniform topology in $[3,14]$ and also different from the perturbation of best response defined in [16]. For example, the existence of essential sets of solutions for VQEP in topological vector spaces is proved in [3], and the uniform metric for two VQEP $\phi_{1}=\left(X, X, C, \varphi_{1}, G_{1}\right)$ and $\phi_{2}=\left(X, X, C, \varphi_{2}, G_{2}\right)$ is defined as

$$
\begin{aligned}
\rho^{\prime \prime}\left(\phi_{1}, \phi_{2}\right)= & \sup _{(x, y) \in X \times X}\left\|\varphi_{1}(x, y)-\varphi_{2}(x, y)\right\| \\
& +\sup _{x \in X} h\left(G_{1}(x), G_{2}(x)\right),
\end{aligned}
$$

where $X$ is a compact convex subset of a Banach space. Naturally, the feasible mapping $G$ requires closed values, which is not a requirement in Theorem 14, however, where each inverse image being open is necessary.

By Theorem 18, each connected component including a connected minimal essential set of solutions is essential; that is, the existence of essential components can be induced.
Corollary 20. Let $\phi \in M^{\prime}$. There is an essential component in $F^{\prime}(\phi)$. If $F^{\prime}(\phi)=\left\{x^{*}\right\}$ is a singleton, then $x^{*}$ is an essential solution of $\phi$.

\section{Acknowledgments}

This project is supported by Guangxi Natural Science Foundation (2012GXNSFBA053013, 2013GXNSFBA19004), NNSF (61164020), and Doctoral Research Fund of GUT.

\section{References}

[1] B.-S. Lee, M. F. Khan, and Salahuddin, "Generalized vector variational-type inequalities," Computers \& Mathematics with Applications, vol. 55, no. 6, pp. 1164-1169, 2008.

[2] S. A. Khan, "Generalized vector complementarity-type problems in topological vector spaces," Computers \& Mathematics with Applications, vol. 59, no. 11, pp. 3595-3602, 2010.

[3] H. Yang and J. Yu, "Essential solutions and essential components of solution set of vector quasi-equilibrium problems," Journal of Systems Science and Mathematical Sciences, vol. 24, no. 1, pp. 7484, 2004.

[4] A. P. Farajzadeh and A. Amini-Harandi, "On the generalized vector equilibrium problems," Journal of Mathematical Analysis and Applications, vol. 344, no. 2, pp. 999-1004, 2008.

[5] C. Finet and L. Quarta, "Vector-valued perturbed equilibrium problems," Journal of Mathematical Analysis and Applications, vol. 343, no. 1, pp. 531-545, 2008.

[6] S. H. Hou, X. H. Gong, and X. M. Yang, "Existence and stability of solutions for generalized strong vector equilibrium problems with trifunctions," Journal of Optimization Theory and Applications, vol. 146, no. 2, pp. 387-398, 2010.

[7] Y.-C. Lin, P.-J. Cheng, and S.-L. Lee, "The convergent behavior for parametric generalized vector equilibrium problems," Journal of Applied Mathematics, vol. 2012, Article ID 183040, 12 pages, 2012.

[8] Z. Lin and J. Yu, "The existence of solutions for the system of generalized vector quasi-equilibrium problems," Applied Mathematics Letters, vol. 18, no. 4, pp. 415-422, 2005.

[9] Q. H. Ansari, "Existence of solutions of systems of generalized implicit vector quasi-equilibrium problems," Journal of Mathematical Analysis and Applications, vol. 341, no. 2, pp. 1271-1283, 2008.

[10] X.-J. Long, N.-J. Huang, and K.-I. Teo, "Existence and stability of solutions for generalized strong vector quasi-equilibrium problem," Mathematical and Computer Modelling, vol. 47, no. 34, pp. 445-451, 2008.

[11] J. Salamon and M. Bogdan, "Closedness of the solution map for parametric weak vector equilibrium problems," Journal of Mathematical Analysis and Applications, vol. 364, no. 2, pp. 483491, 2010.

[12] K. Kimura and J.-C. Yao, "Sensitivity analysis of solution mappings of parametric vector quasi-equilibrium problems," Journal of Global Optimization, vol. 41, no. 2, pp. 187-202, 2008.

[13] L. Q. Anh and P. Q. Khanh, "Continuity of solution maps of parametric quasiequilibrium problems," Journal of Global Optimization, vol. 46, no. 2, pp. 247-259, 2010.

[14] X. Fan, C. Cheng, and H. Wang, "Stability analysis for vector quasiequilibrium problems," Positivity, 2012. 
[15] Q. H. Ansari, S. Schaible, and J.-C. Yao, "The system of generalized vector equilibrium problems with applications," Journal of Global Optimization, vol. 22, no. 1-4, pp. 3-16, 2002.

[16] Z. Lin, H. Yang, and J. Yu, "On existence and essential components of the solution set for the system of vector quasiequilibrium problems," Nonlinear Analysis, Theory, Methods and Applications, vol. 63, no. 5-7, pp. e2445-e2452, 2005.

[17] Z. Yang and Y. J. Pu, "On existence and essential components for solution set for system of strong vector quasi-equilibrium problems," Journal of Global Optimization, vol. 55, no. 2, pp. 253-259, 2013.

[18] Q. Q. Song and L. S. Wang, "The existence of solutions for the system of vector quasi-equilibrium problems in topological order spaces," Computers \& Mathematics with Applications, vol. 62, no. 4, pp. 1979-1983, 2011.

[19] S. Plubtieng and K. Sitthithakerngkiet, "On the existence result for system of generalized strong vector quasiequilibrium problems," Fixed Point Theory and Applications, vol. 2011, Article ID 475121, 9 pages, 2011.

[20] C. D. Horvath and J. V. Llinares Ciscar, "Maximal elements and fixed points for binary relations on topological ordered spaces," Journal of Mathematical Economics, vol. 25, no. 3, pp. 291-306, 1996.

[21] D. T. Luc, Theory of Vector Optimization, Springer, Berlin, Germany, 1989.

[22] A. Capătă and G. Kassay, "On vector equilibrium problems and applications," Taiwanese Journal of Mathematics, vol. 15, no. 1, pp. 365-380, 2011.

[23] J. Yu and Y. H. Zhou, "A Hausdorff metric inequality with applications to the existence of essential components," Nonlinear Analysis. Theory, Methods \& Applications, vol. 69, no. 5-6, pp. 1851-1855, 2008. 


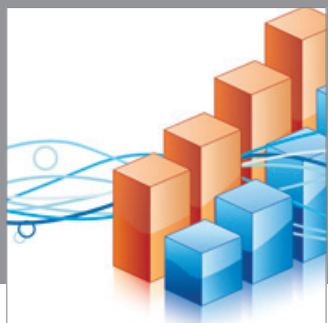

Advances in

Operations Research

mansans

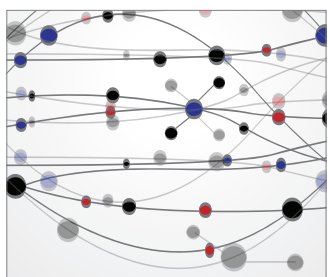

The Scientific World Journal
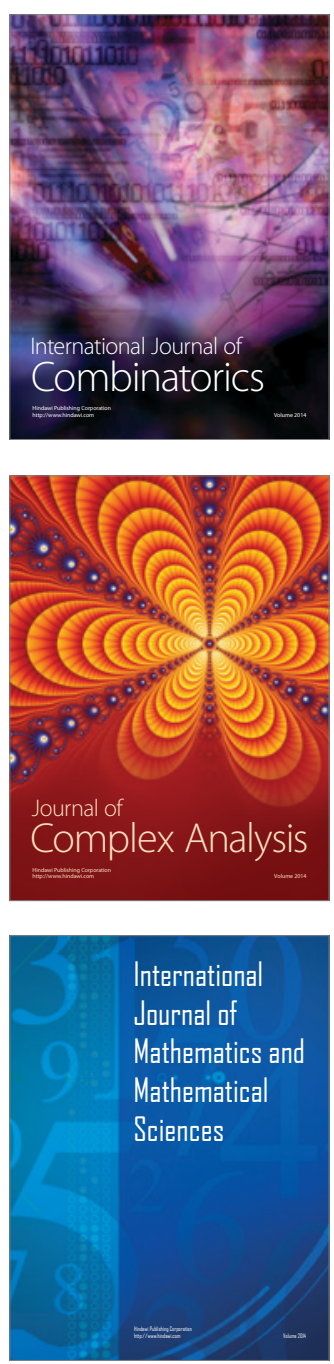
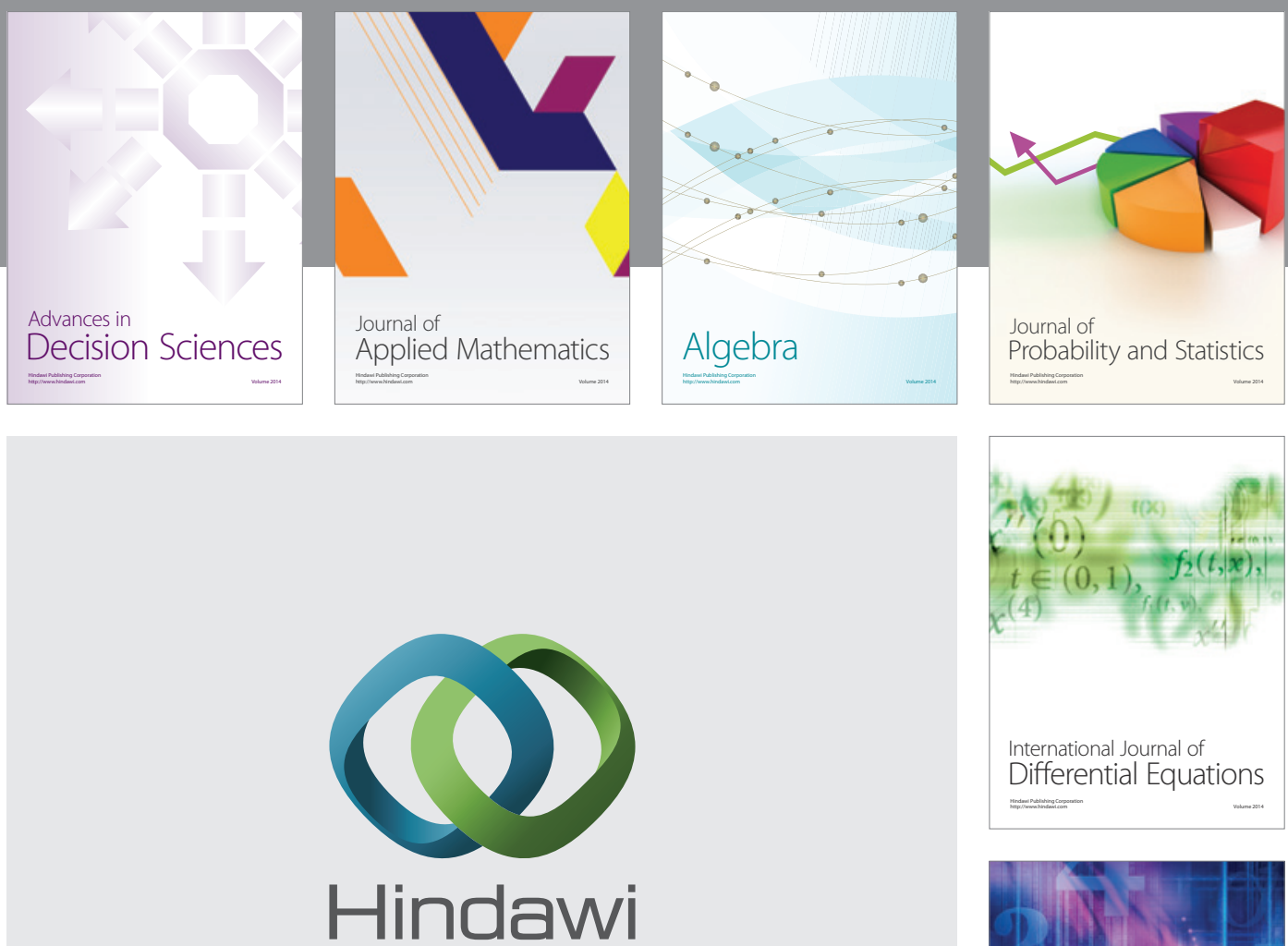

Submit your manuscripts at http://www.hindawi.com
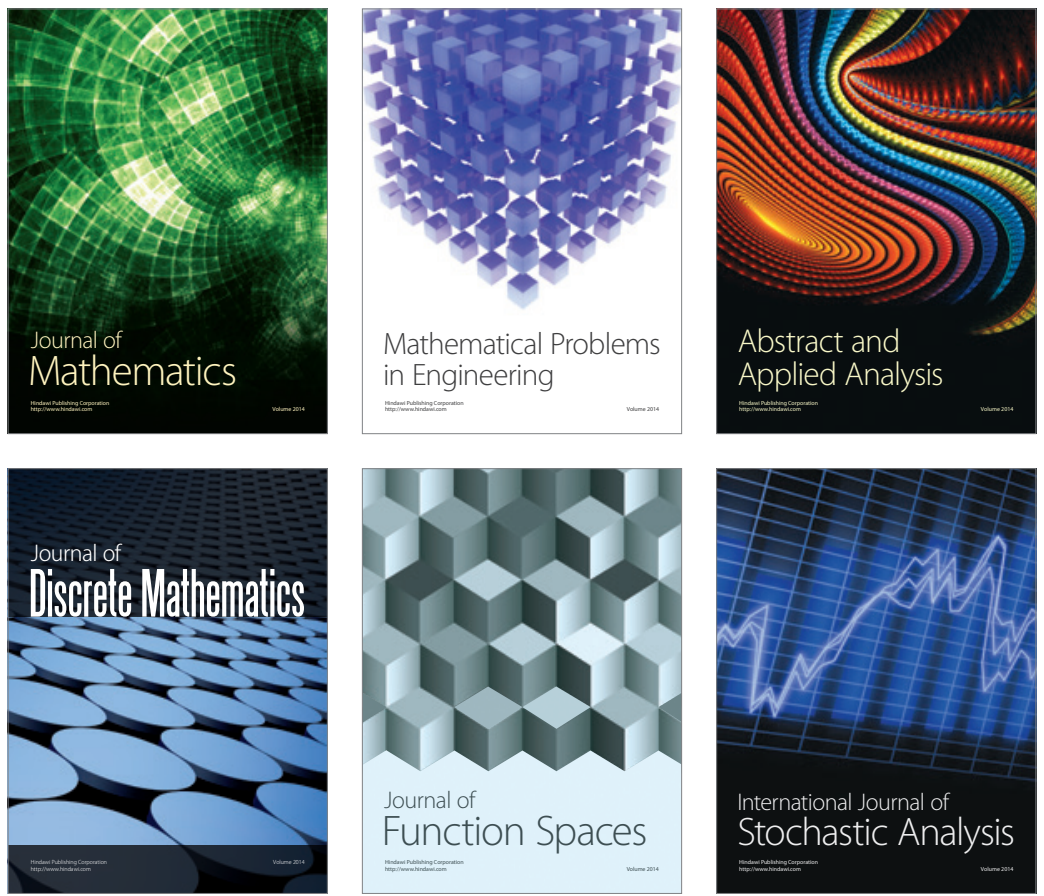

Journal of

Function Spaces

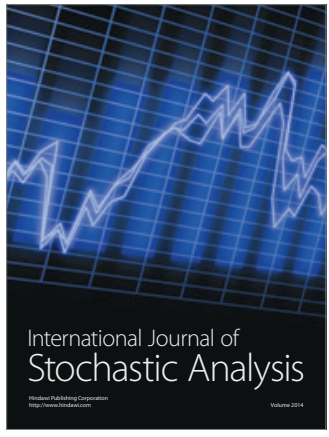

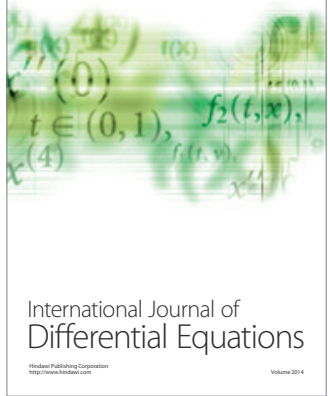
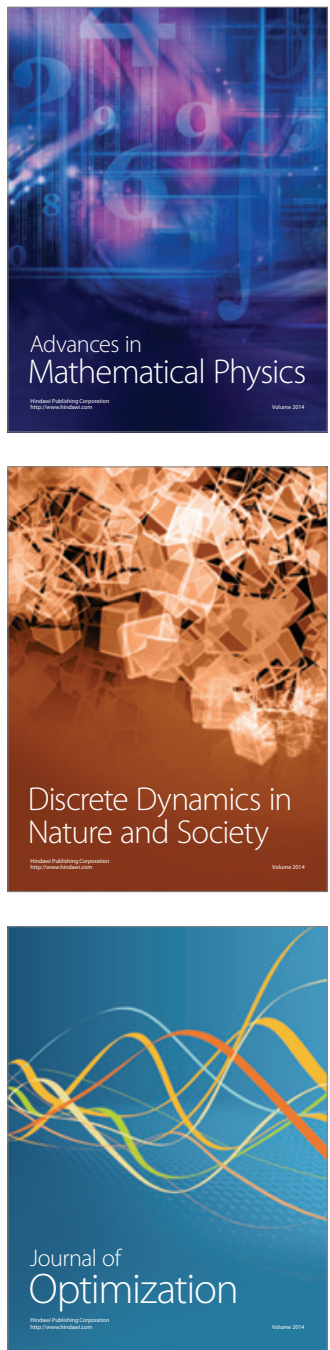